\title{
Laparoscopic Transabdominal Preperitoneal Mesh Hernioplasty: A Medical College Experience
}

\author{
Mushtaq Chalkoo*, Mujahid Ahmad Mir, Hilal Makhdoomi \\ Department of General Surgery, Government Medical College, Srinagar, India \\ Email: "mushtaq_chalkoo@rediffmail.com
}

Received 28 January 2016; accepted 22 February 2016; published 25 February 2016

Copyright (C) 2016 by authors and Scientific Research Publishing Inc.

This work is licensed under the Creative Commons Attribution International License (CC BY). http://creativecommons.org/licenses/by/4.0/

(c) (i) Open Access

\begin{abstract}
Objectives: To determine the feasibility and patient's outcome of laparoscopic transabdominal preperitoneal mesh hernioplasty for inguinal hernias. Patients and Methods: This study was carried out from March 2011 to April 2014. A total of 130 patients underwent laparoscopic transabdominal preperitoneal mesh hernioplasty (TAPP) for uncomplicated inguinal hernia. Of this, 10 patients presenting with bilateral inguinal hernias were operated in the single sitting. A $15 \mathrm{~cm} \times$ $12 \mathrm{~cm}$ polypropylene mesh was used in all cases. Operative morbidity, postoperative pain, seroma formation, evidence of superficial infection, chronic groin pain and hernia recurrence were noted. The majority of the patients were discharged within 24 hours and follow-up was done at 1 week, 1 month, and 6 months. Results: 130 patients presenting with uncomplicated inguinal hernias were operated over a period of three years in the department of surgery, Govt. Medical College Srinagar. The mean age of the patients was 39.18 years (range: 18 - 70 years). The median duration of operation was 48.5 minutes (range: 18 - 120 minutes). None of the procedure was converted to open inguinal hernia repair. Postoperative pain was observed in $9.23 \%$ of the cases and was easily controlled by oral analgesics. Six patients $(4.62 \%)$ developed seroma, out of which one required aspiration while others settled conservatively. Two patients $(1.54 \%)$ developed wound infection and one patient $(0.77 \%)$ had recurrence. None of the patients developed scrotal hematoma or neuralgia. Return to normal activity after TAPP repair was found to be after a median of 16.1 days. Conclusion: Transabdominal preperitoneal repair for inguinal hernia using proline mesh may be a safe and effective procedure with low morbidity, early return to normal activity and with a very low recurrence after six months follow-up.
\end{abstract}

\section{Keywords}

Inguinal Hernias, Laparoscopy, Transabdominal Preperitoneal Hernia Repair, Feasibility,

\footnotetext{
${ }^{*}$ Corresponding author.
} 


\section{Complications}

\section{Introduction}

Minimal access surgical strategy for inguinal hernia repair was added to the procedural armamentarium in early 1990s as laparoscopy began to establish its foothold in general surgery [1]. Inguinal hernias account for $75 \%$ of all abdominal wall hernias, and with a lifetime risk of $27 \%$ in men and $3 \%$ in women [2] [3]. Laparoscopic inguinal hernia repair includes: totally extraperitoneal (TEP) repair; transabdominal preperitoneal (TAPP) repair or intraperitoneal onlay mesh (IPOM) repair. Unlike laparoscopic cholecystectomy, which was very quickly accepted by the surgeons worldwide, laparoscopic hernia repair faced a stumbled welcome. Laparoscopic hernia repair is technically more demanding than its open counterpart and there is evidence of a long learning curve [4].

Compared with open repairs, laparoscopic inguinal hernia repair has some advantages, including less postoperative pain, a shorter recovery period, earlier return to daily activities and work, better cosmetic results and decreased recurrence rate. A number of studies have shown laparoscopic repair of inguinal hernias to have advantages over conventional repair [5]-[7]. The laparoscopic repair has some disadvantages as well, which includes cost and steep learning curve. These advantages continued to fuel the progression of laparoscopic hernia repair. We took up this study to prospectively analyse the feasibility and patient outcome of laparoscopic transabdominal preperitoneal hernioplasty for the management of uncomplicated inguinal hernias. The laparoscopic TAPP works on Pascal's law of hydraulics.

\section{Aims and Objectives}

To determine the feasibility and patient's outcome of laparoscopic transabdominal preperitoneal mesh hernioplasty for inguinal hernias.

\section{Materials and Methods}

The study was conducted in the Post-Graduate Department of General Surgery Government Medical College Srinagar from March 2011 to April 2014. It was a prospective, observational study on the patients of uncomplicated inguinal hernias who underwent an elective laparoscopic inguinal hernia repair. The approval from the ethics committee and a signed informed consent were obtained from the patients. All patients, above 18 years of age with uncomplicated inguinal hernia were included in the study. Complicated hernias, patients unfit for general anaesthesia and patients with previous lower abdominal or pelvic surgery were excluded. Operations were undertaken by a single surgeon of our team and all patients were followed up for a period of six months post operatively. The following data was collected prospectively: age, sex, duration of surgery, intra-operative complications, postoperative complications, hospital stay and recurrence. All the patients enrolled for the study were evaluated by detailed history, thorough general physical examination, and focussed systemic examination. Informed consent was taken before surgery in the language the patients understood. All patients received a prophylactic dose of injection ceftriaxone $1 \mathrm{~g}$ half an hour before surgery.

Operative procedure: All patients were asked to void before the start of the procedure. General anaesthesia with endotracheal intubation was preferred. The surgeon stood on the contralateral side of the hernia. The head end of the table was slanted down to $15^{\circ}$ for the establishment of pneumoperitoneum by closed technique. A 10 $\mathrm{mm}$ optical port was then placed through the supra-umbilical incision. Two $5 \mathrm{~mm}$ ports were placed as working ports for the right and left hand of the surgeon, one on each side, at the level of the umbilicus in the midclavicular line. The hernia was inspected and its type (direct or indirect) established by the position of the defect with respect to the inferior epigastric vessels and cord structures (Figure 1). Contents of the hernial sac, if any, were reduced with the help of atraumatic bowel forceps. The peritoneal incision was started at a point midway between the groin crease and the umbilicus, generally about $2 \mathrm{~cm}$ above the internal ring (Figure 2). It extended from above the anterior superior iliac spine to the medial umbilical ligament. The flap was raised by both blunt and sharp dissection from the cephalic to caudal direction (Figure 3). It was easier to raise only the lower flap when compared with raising a lower along with an upper flap. The dissection was continued medially to the symphysis pubis to visualize the space of Retzius. After the medial dissection, the flap was raised lateral to the 


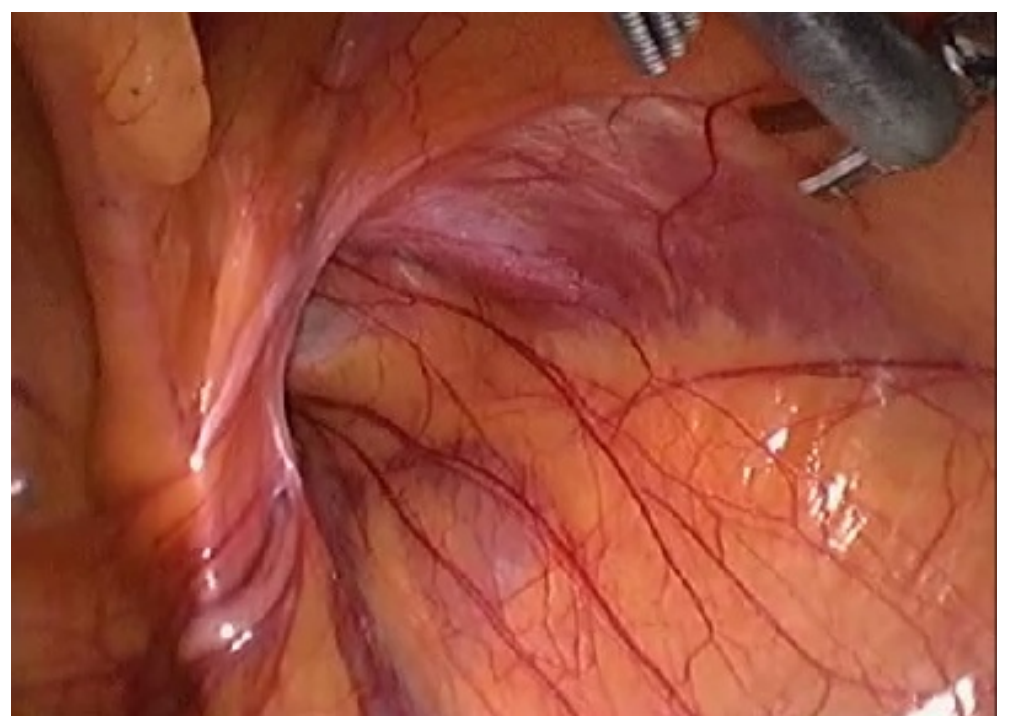

Figure 1. Shows laparoscopic view of patulous /defective inguinal ring.

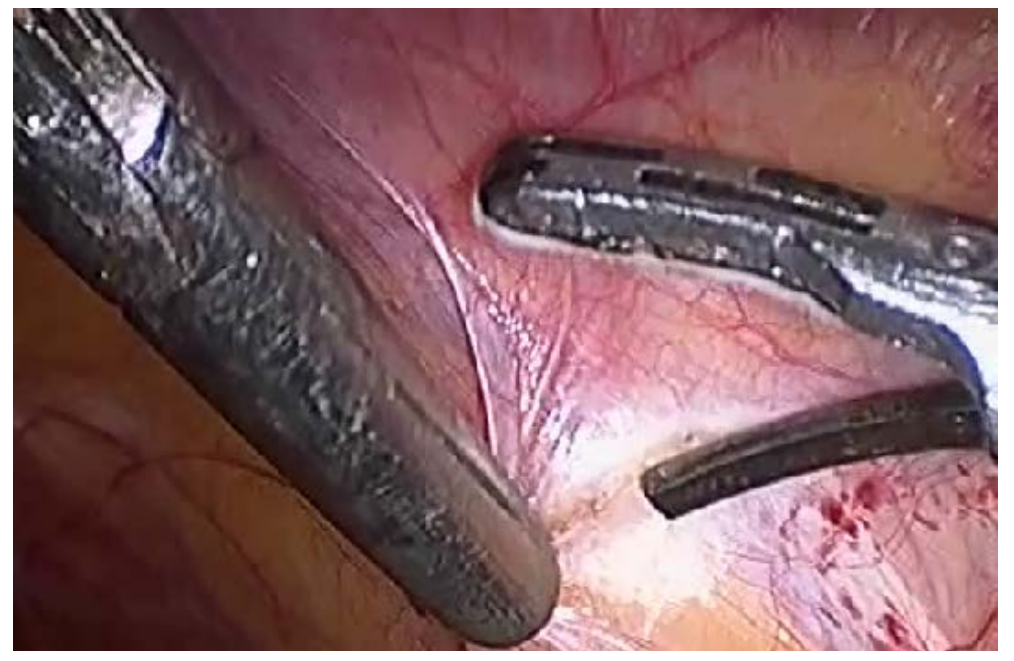

Figure 2. Showing dissection at $2 \mathrm{~cm}$ superolateral to deep inguinal ring.

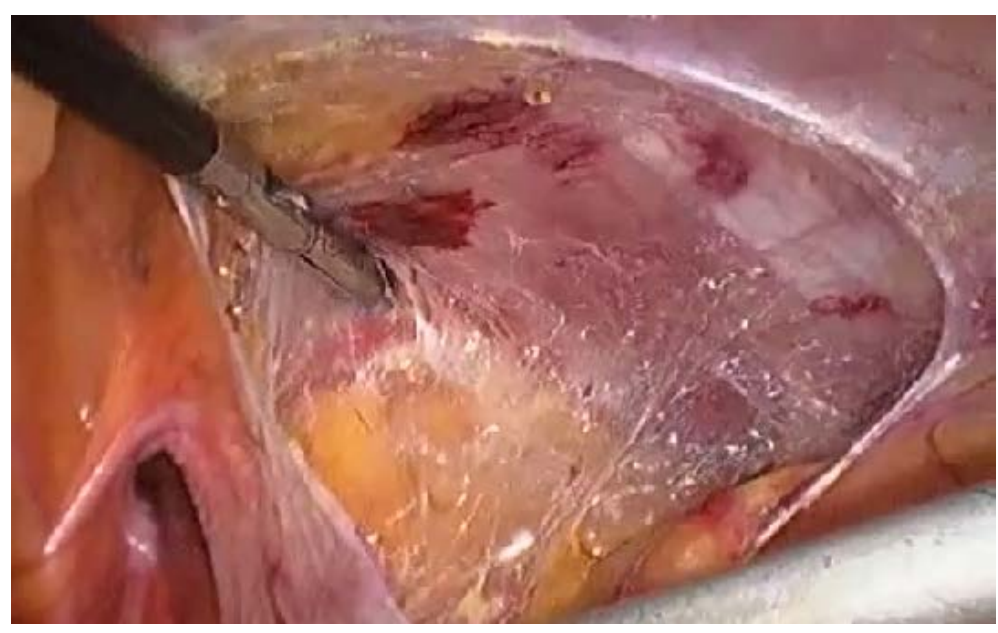

Figure 3. Development of lateral pre-peritoneal pouch. 
internal ring up to the anterior superior iliac spine. The hernial sac lies anterior and lateral to the cord structures (Figure 4). The dissection of the sac was performed and obliterated by vicryl endoloop (Figure 5). A polypropylene mesh of $15 \mathrm{~cm} \times 12 \mathrm{~cm}$ was used for the repair (Figure 6). The mesh was introduced into the operating field through the $10 \mathrm{~mm}$ umbilical port by removing the telescope, after which the telescope was reinserted. Staples were applied over the medial and upper border of the mesh to anchor it to the underlying muscles. Generally three staples were sufficient: one on the medial border and two on the upper border. After placement of the mesh, the peritoneal flap was closed over the mesh to prevent bowel and omental adhesions; this was done with sutures (Figure 7). The carbon dioxide gas was evacuated to empty the abdominal cavity and scrotum. The ports were removed after lifting the anterior abdominal wall. The sheath of the $10 \mathrm{~mm}$ port was closed. Skin incisions were closed with simple sutures. In cases of bilateral hernias, we used two separate pieces of mesh that were secured together in the midline. The mesh was placed in the first hernia but the peritoneum was not closed until the other side was completed.

\section{Postoperative Care}

- Orals were started as soon as the patients started to tolerate them.

- A second dose of intravenous antibiotics (Ceftriaxone $1 \mathrm{~g}$ IV) was given 12 hours after surgery.

- Analgesics were given on demand only.

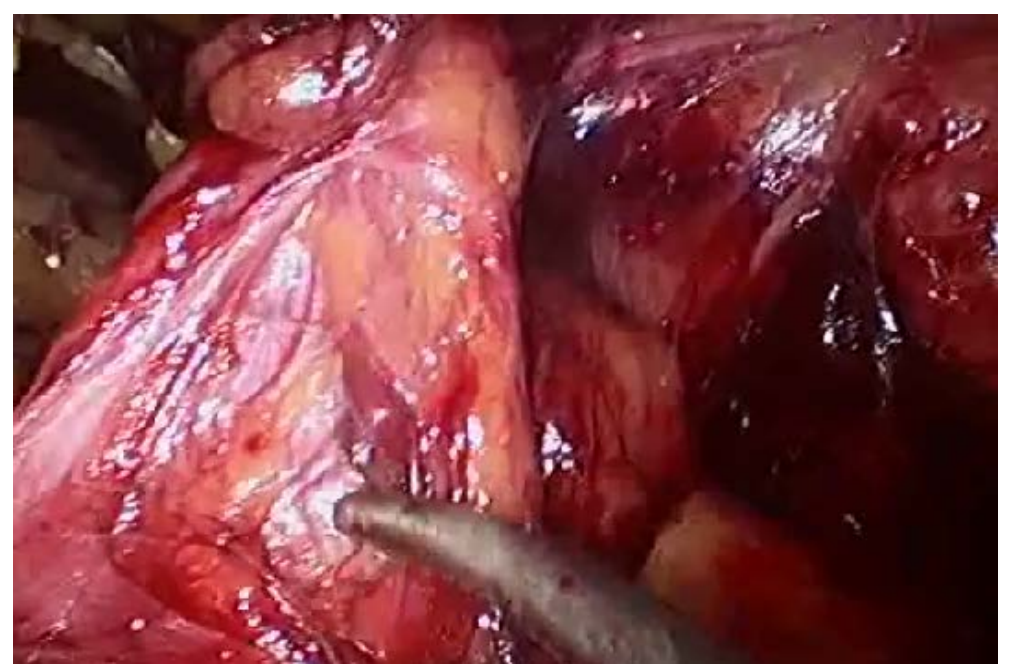

Figure 4. Showing separation of sac from cord structures.

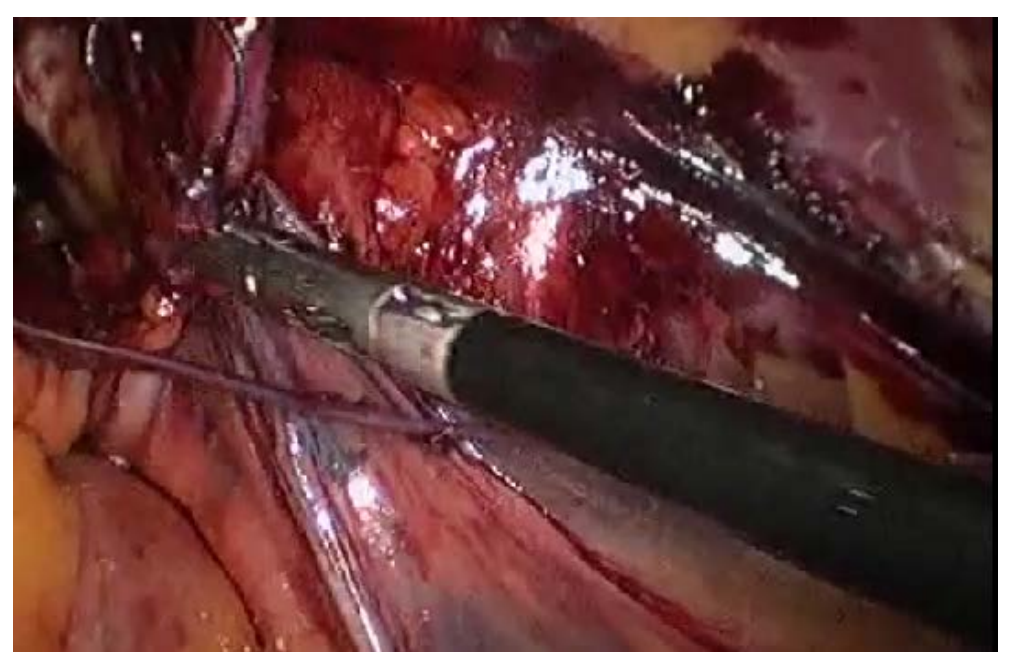

Figure 5. Showing endolooping of sac by vicryl. 


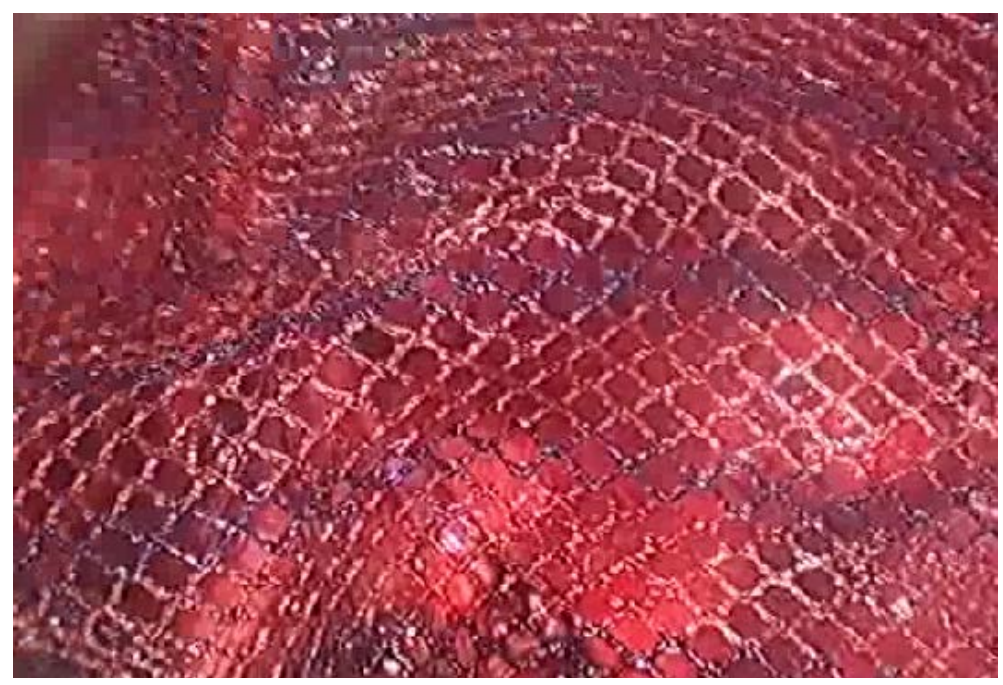

Figure 6. Showing display of prolene mesh.

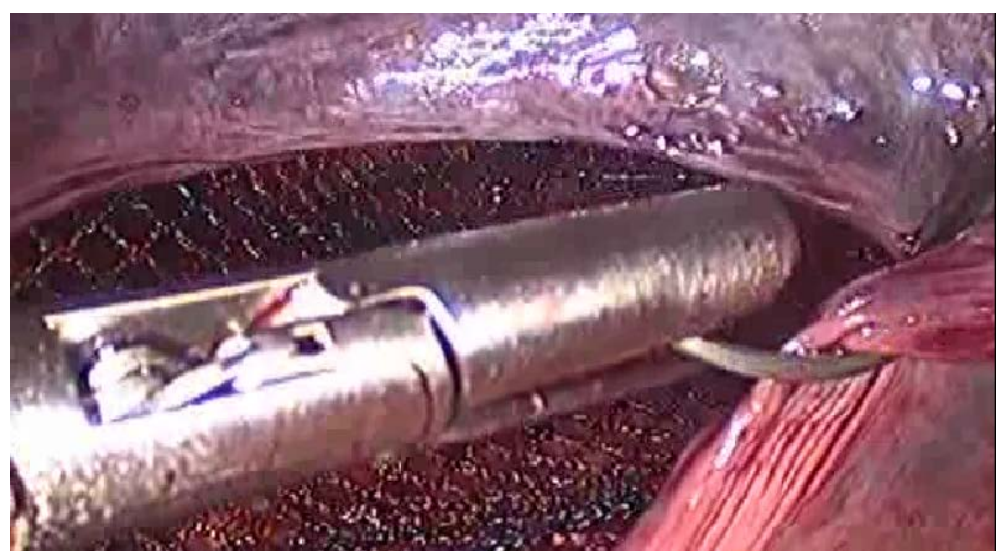

Figure 7. Showing V-lock used for repair of peritoneum.

Monitoring of the patient for Pulse, Blood pressure, Temperature, Respiratory rate, urine output (color and quantity), and appearance of bowel sounds and passage of flatus was done. The patients were typically discharged home on 1st postoperative day on tablet Cefuroxime $500 \mathrm{mg}$ BD and tablet diclofenac $25 \mathrm{mg}$ BD for 3 days orally postoperatively. The patients were instructed to avoid heavy lifting for 3 months postoperatively. Patients were followed in the office at 1 week, 4 weeks and 6 months.

\section{Results}

A total of 130 patients were operated during the study period and the total number of hernias repaired were 140; 10 patients had bilateral hernia repair. Patients included in the study were adults and their ages ranged from 18 to 70 years with a mean age of 39.17 years. Most of the patients were 40 to 49 years of age, 43 patients (33.04\%). 5 patients (3.85\%) were below the age of 20 years. Patients over the age of 60 years were 11 (8.46\%) (Table 1).

There were only 5 (3.85\%) female patients in our study with one of them having bilateral hernia. Out of 130 patients 10 had bilateral hernias. Majority of the patients in our study had indirect hernias (as shown in Table 2).

The median duration of operation was 48.5 minutes (range 18 - 120 minutes) for TAAP repair. It was observed that with experience, the learning curve for the laparoscopic inguinal hernia repair shortened that is from the first case which took 120 minutes the duration decreased to 18 minutes in a few later cases of laparoscopic repair. The main complications in our study were post-operative pain, seroma formation and port site infection (as shown in Table 3 ). 
Table 1. Age distribution $(\mathrm{n}=130)$.

\begin{tabular}{ccc}
\hline Age group & Number of patients & Percentage \\
\hline$<20$ years & 5 & $3.85 \%$ \\
$20-29$ years & 36 & $27.75 \%$ \\
$30-39$ years & 21 & $16.02 \%$ \\
$40-49$ years & 43 & $33.04 \%$ \\
$50-59$ years & 14 & $10.77 \%$ \\
60 years and above & 11 & $8.46 \%$ \\
\hline
\end{tabular}

Age $($ mean $\pm \mathrm{SD})=39.18 \pm 3.01$.

Table 2. Clinical diagnosis $(\mathrm{n}=140)$.

\begin{tabular}{cccc}
\hline Type of hernia & & Subjects & Percentage \\
\hline \multirow{2}{*}{ Indirect inguinal hernia } & Right & 45 & $32.14 \%$ \\
& Left & 37 & $26.43 \%$ \\
\multirow{2}{*}{ Direct inguinal hernia } & Right & 32 & $22.86 \%$ \\
& Left & 26 & $18.57 \%$ \\
Bilateral inguinal hernia & Direct & 06 & $04.29 \%$ \\
& Indirect & 04 & $02.56 \%$ \\
\hline
\end{tabular}

Table 3. Post-operative complications $(n=130)$.

\begin{tabular}{cccc}
\hline S. No. & Postoperative complication & No. of patients & Percentage \\
\hline 1. & Pain & 12 & $9.23 \%$ \\
2. & Seroma & 6 & $4.62 \%$ \\
3 & Port site infection & 2 & $1.54 \%$ \\
4. & Recurrence & 1 & $0.77 \%$ \\
5. & Scrotal hematoma & None & $0 \%$ \\
\hline
\end{tabular}

Majority of our patients were discharged home within 24 hours after the operation. Only 6 patients stayed for 72 hours ( 3 days) and 2 patients stayed for 4 days. Recovery in the community was significantly quicker after TAAP hernia repair. Patients returned to work after a median of 16.1 days (range 5 - 30 days) irrespective of whether the patients were self employed or employees. Similarly, patients required less analgesia at home and returned to their normal lifestyle more rapidly. During follow-up at one month there was no recurrence but at 6 months follow-up, 1 patient reported recurrence.

\section{Discussion}

The surgical history of inguinal hernias dates back to ancient Egypt. From Bassini's heralding of the modern era to today's mesh-based open and laparoscopic repairs, this history parallels closely the evolution in anatomical understanding and development of techniques of general surgery. Although open, mesh-based, tension-free repair remains the criterion standard, laparoscopic herniorrhaphy, in the hands of adequately trained surgeons, produces excellent results comparable to those of open repair. In our study, 33\% patients were in age group of 40 - 49 years. Mean patient age was $39.18 \pm 3.01$ years; comparable with that in the study conducted by Ayman et al. [8] where it was 39.37 years (range 18 - 60). 96.15\% (125/130) patients were males. In the study conducted by Ghani et al. [9] 96.02\% of inguinal hernias were in men. Most common type of hernia seen in our study was unilateral indirect inguinal hernia which comprised of 58.57\% (82/130). Our results are comparable to Ayman et al. [8], who found $52.8 \%$ of patients having unilateral indirect hernia. The median operative time was 48.5 minutes (range 18 - 120 minutes) for TAPP repair. It was observed that with experience, the learning curve for the laparoscopic inguinal hernia repair shortened that is from the first case which took 120 minutes the duration decreased to 38 minutes in a few later cases of laparoscopic repair which is at par with Zeineldin [10] (43 \pm 
11.3). The mean operative duration in TAAP repair was $60.13 \pm 14.76$ minutes in the study by Ghani et al. [9]. Most of patients, 124 (94.9\%) in our study were discharged within 24 hours and returned to routine work in median of 16.1 days. In study by Ayman et al. [8] the mean time to return to work after TAAP was 14.4 days which is comparable to what we have observed in our study. Postoperative pain was seen in 12 (9.23\%) patients which is at par with observation of Sondenaa et al. [11] who reported postoperative pain in $10 \%$ of patients. Furthermore, Zeineldin [10] found seroma in 6\% of patients which matched our observation in 4.62\% (6/130) patients. However they were managed conservatively without aspiration or wound exploration. It was reported that a very smooth, nearly painless postoperative period and a limited number of early postoperative complications compensate for most disadvantages of the laparoscopic method. Even though laparoscopic repair of hernia is frequently regarded as equivalent to classical techniques, it has not been used widely. Several factors account for that. The procedure is technically more demanding than the open inguinal operation and it must be performed under general anaesthesia. The cost of instruments, mesh and clip appliers is another important drawback. The current study may see more recurrences in a longer follow-up. However, the excellent results, a low complication rate, and a smooth, nearly painless postoperative course are very encouraging, and in many centres specializing in laparoscopic surgery these procedures are common. Because the laparoscopic approach has shown clear advantages regarding less chronic postoperative pain and numbness, fast return to normal activities, and a decrease in the incidence of wound infection and hematoma, it should be considered an appropriate approach for inguinal hernia surgery.

\section{Conclusion}

We conclude that laparoscopic hernia repair may be a safe and effective alternative to open inguinal hernia repair which enables a faster convalescence and return to productive activity, with fewer complications and a recurrence rate as low as that of an open mesh repair. The limitations of the study are a short follow-up. Laparoscopic hernia repair does require special equipment and training and the learning curve of the procedure is long but the operating time reduces with experience.

\section{References}

[1] Ger, R., Mishrick, A., Hurwitz, J., Romero, C. and Oddsen, R. (1993) Management of Groin Hernias by Laparoscopy. World Journal of Surgery, 17, 46-50. http://dx.doi.org/10.1007/BF01655704

[2] Javid, P.J. and Brooks, D.C. (2007) Hernias. In: Zinner, M.J. and Ashley, S.W., Eds., Maingots Abdominal Operations, Vol. 1, 11th Edition, McGraw-Hill, New York, 103-139.

[3] Gulzar, M.R., Iqbal, J., Ulhaq, M.I. and Afzal, M. (2007) Darning vs Bassini Repair for Inguinal Hernia—A Prospective Comparative Study. Professional Medical Journal, 14, 128-133.

[4] Wright, D. (1998) The Learning Curve for Laparoscopic Hernia Repair. Seminars in Laparoscopic Surgery, 5, $227-$ 232. http://dx.doi.org/10.1177/155335069800500405

[5] Memon, M.A., Cooper, N.J., Memon, B., Memon, M.I. and Abrams, K.R. (2003) Metaanalysis of Randomized Clinical Trials Comparing Open and Laparoscopic Inguinal Hernia Repair. British Journal of Surgery, 90, 1479-1492. http://dx.doi.org/10.1002/bjs.4301

[6] McCormack, K., Scott, N., Go, P.M., Ross, S.J. and Grant, A. (2003) Collaboration the EU Hernia Trialists: Laparoscopic Techniques versus Open Techniques for Inguinal Hernia Repair. Cochrane Database of Systematic Reviews, 2003, CD001785.

[7] Takata, M.C. and Duh, Q.Y. (2008) Laparoscopic Inguinal Hernia Repair. Surgical Clinics of North America, 88, 157178. http://dx.doi.org/10.1016/j.suc.2007.10.005

[8] Elwan, A.M., Abomera, M.A., Abo Al Makarem, M.A., and Abd Alhamed, H. (2013) Mohammedain: Laparoscopic Transabdominal Preperitoneal Repair versus Open Preperitoneal Mesh Repair for Inguinal Hernia. Journal of the Arab Society for Medical Research, 8, 38-42.

[9] Ghani, A., Khalil, J., Khan, M.I. and Khan, H. (2012) Laparoscopic Transabdominal Preperitoneal versus Lichtenstein Tension Free Repair for Inguinal Hernia. Pakistan Journal of Surgery, 28, 6-11.

[10] Zeineldin, A. (2008) Transabdominal (TAPP) versus Total Extraperitoneal (TEP) Laparoscopic Inguinal Hernia Repair: A Prospective Comparative Study. Menoufiya Medical Journal, 21.

[11] Sondenaa, K., Nesvik, I., Breivik, K. and Korner, H. (2001) Long-Term Follow up of 1059 Consecutive Primary and Recurrent Inguinal Hernias in a Teaching Hospital. European Journal of Surgery, 67, 125-129. 